\title{
Enhancing dissolution profile of diazepam using hydrophilic polymers by solid dispersion technique
}

Md. Sariful Islam Howlader ${ }^{1}$, Jayanta Kishor Chakrabarty ${ }^{2},{ }^{*} \mathrm{Khandokar} \mathrm{Sadique} \mathrm{Faisal}^{2}$, Uttom Kumar ${ }^{3}$, Md. Raihan Sarkar ${ }^{3}$, Mohammad Firuz Khan ${ }^{3}$

${ }^{1}$ Department of Pharmacy, World University of Bangladesh, Dhanmondi, Dhaka 1205, Bangladesh

${ }^{2}$ Department of Pharmacy, Northern University of Bangladesh, Kazi Nazrul Islam Avenue, Dhaka-1215, Bangladesh

${ }^{3}$ Faculty of Pharmacy, University of Dhaka, Dhaka 1000, Bangladesh

\begin{abstract}
The aim of the present study was to improve the solubility and dissolution rate of a poorly water-soluble drug by a solid dispersion technique, in order to investigate the effect of these polymers on release mechanism from solid dispersions. Diazepam was used as a model drug to evaluate its release characteristics from different matrices. Solid dispersions were prepared by using polyethylene glycol 6000 (PEG-6000), HPMC, HPC and Poloxamer in different drug-to-carrier ratios (1:2, 1:4, 1:6, 1:8, 1:10). The solid dispersions were prepared by solvent method. The pure drug and solid dispersions were characterized by in vitro dissolution study. Distilled water was used as dissolution media, $1000 \mathrm{ml}$ of distilled water was used as dissolution medium in each dissolution basket at a temperature of $37^{\circ} \mathrm{C}$ and a paddle speed of $100 \mathrm{rpm}$. The very slow dissolution rate was observed for pure Diazepam and the dispersion of the drug in the polymers considerably enhanced the dissolution rate. This can be attributed to improved wettability and dispersibility, as well as decrease of the crystalline and increase of the amorphous fraction of the drug. SEM (Scanning Electron microscope) studies shows that the solid dispersion having a uniform dispersion. Solid dispersions prepared with PEG-6000, Poloxamer showed the highest improvement in wettability and dissolution rate of Diazepam. Solid dispersion containing polymer prepared with solvent method showed significant improvement in the release profile as compared to pure drug, Diazepam.
\end{abstract}

Key Words: Molecular dispersion, hydrophilic polymer, SEM, Solvent evaporation technique, gamma-aminobutyric acid.

\section{INTRODUCTION}

In drug development, enhancing the oral bioavailability of poor water soluble drugs remains most challenging aspects. The dispersion of an active ingredient in an inert hydrophilic carrier at solid form prepared by fusion, solvent evaporation technique is defined as solid dispersion (Sadika and Ismail, 2012) Solid dispersions (SDs) traditionally have been used as an effective method to improve the dissolution properties and bioavailability of poorly water-soluble drugs. Molecular dispersion of the drug in polymeric carriers may lead to particle size reduction and surface area enhancement, which

*Corresponding Author:

Khandokar Sadique Faisal, Assistant Lecturer

Department of Pharmacy, Northern University Bangladesh

Kazi Nazrul Islam Avenue

Dhaka-1215, Bangladesh

E-mail:khandokar.sadique.faisal@gmail.com

Contact No.: 08801912958959 result in improved the dissolution rates. Furthermore, no energy is required to break up the crystal lattice of a drug during the dissolution process, improvement in the drug solubility and wettability due to surrounding hydrophilic carriers also result in increased dissolution of poorly water soluble drug (Yamashtia et al., 2003).

Recently many potential drug candidates have been identified using advance molecular screening method. It is a common fact that though having a potential therapeutic effect, many drugs are abandoned due to poor aqueous solubility. Solid dispersion technique may be useful for solving the solubility and bioavailability problem of such drugs (Leuner et al., 2000). By definition, solid dispersions are formulations of finely crystalline or amorphous drug dispersed in an inert matrix (Chio and Rigelman, 1971). 
Reduction or absence of aggregation and agglomeration may also contribute to increase dissolution. Methods used to prepare solid dispersions include the melting method, the solvent method, and the solvent wetting method (Mooter et al., 1998; YhNam et al., 2000).

Among the various approaches employed to improve the dissolution of poorly soluble drugs, solid dispersion has been proven successful. Fast or immediate drug dissolution from solid dispersions has been observed due to increased wettability, improved dispersibility of drug particles, the existence of the drug in amorphous form with improved solubility and absence of aggregation of drug particles (Gohel et al., 2002). Literature shows that the solvent evaporation method has been used for the preparation of solid dispersions for dissolution enhancement (USP, 1995).

Polymeric carriers have been the most successful for solid dispersions, because they are able to originate amorphous solid dispersions. They are divided into fully synthetic polymers and natural polymers. Synthetic polymers include povidone (PVP), polyethyleneglycols (PEG) and polymethacrylates . Natural polymers are mainly composed of cellulose derivatives, such as hydroxypropylmethylcellulose (HPMC), ethylcellulose or starch derivates, like cyclodextrins. Amorphous solid dispersions can be classified according to the molecular interaction of drugs and carriers in solid solutions, solid suspensions or a mixture of both. In amorphous solid solutions, drug and carrier are totally miscible and soluble which originating a homogeneous molecular interaction between them. In these systems, the drug and carrier interaction energy is extremely high, resulting in a really true solution (Thahera et al., 2012; Costantino et al., 2002). In the present experiment Diazepam belongs to a class of anticonvulsants that enhances gamma-aminobutyric acid (GABA) receptor responses. Anticonvulsants used for several types of seizures, including myotonic or atonic seizures, photosensitive epilepsy, and absence seizures. Diazepam exerts its action by binding to the benzodiazepine site of the GABA receptors, which causes an enhancement of the electric effect of GABA binding on neurons resulting in an increased influx of chloride ions into the neurons. This results in an inhibition of synaptic transmission across the central nervous system
(Skerritt et al., 1983; Lehoullier et al., 1987). Diazepam is a light yellow crystalline powder which is practically odorless. It is freely very soluble in methanol, ethanol, and acetone, and practically insoluble in water (at $25^{\circ} \mathrm{C}<0.1 \mathrm{mg} / \mathrm{ml}$ ). It is generally considered that compounds with a very low aqueous solubility will show dissolution rate-limited absorption and, hence, poor absorption, distribution, and target organ delivery (Proudfoot et al., 1991). Improvement of aqueous solubility in such a case is a valuable goal to improve therapeutic efficacy.

Several attempts have been reported in literature using solid dispersion technique to increase the dissolution characteristics where Hydroxy Propyl methyl cellulose (HPMC), polyvinylpyrrolidone (PVP), polyethylene glycol, sodium carboxymethyl cellulose, sodium starch glycolate, pregelatinized starch were used as solubilizing agents (Douroumis et al., 2007; Nair et al., 2002; Rane et al., 2007). In this study solid dispersions were formulated with three water soluble polymers by solvent evaporation technique. Micronized poloxamer 407, HPMC 6 cps and HPC were utilized for this purpose. Poloxamers are nonionic polyoxyethylene-poly-oxypropylene copolymers used primarily as emulsifiers, solubilizing agents, wetting agents and have been reported for enhancing the solubility and bioavailability of sparingly soluble drugs in solid dosage forms (Chen et al., 2004; Passerini et al., 2002; Yu et al., 2007). Solid dispersions were prepared with poloxamer by melting method for rofecoxib and ibuprofen (Newa et al., 2008). Reduced crystalline structure and improved wet ability, (Shah et al., 2007; Chokshi et al., 2007) were mentioned as the mechanism by which poloxamer can enhance dissolution from solid dispersions. On the other hand, both HPMC and PVP are known to act as crystallization inhibitors and thus they help to produce solid solutions (Konno et al., 2008; Gao et al., 2003; Iervolino et al., 2001; Hisu-O et al., 1996). They improve the dissolution characteristics due to interaction through hydrogen bonding.

The objective of the present study was to improve the solubility of Diazepam by using three different types of water soluble polymers. The formulations were characterized by in vitro dissolution study to compare the effects of polymers on the preparation of solid dispersion and dissolution enhance. 


\section{MATERIALS AND METHODS}

\section{Materials}

Diazepam was a gift sample from Rangs Pharmaceuticals Ltd., Dhaka, Bangladesh. Poloxamer 407 (Lutrol 127) was gifted by BASF, Germany. HPMC 6 cps (Shin Etsu Chemical Company Ltd., Japan), HPC (Samsung, Korea) and reagent grade methanol (Merck, Germany) were purchased from the market.

\section{Preparation of solid dispersions}

The solvent process either comprises dissolving a sparingly water-soluble drug and a water-soluble polymer, i.e. the carrier, in an organic solvent capable of dissolving both and removing the solvent by evaporation or comprises dissolving the drug in an organic solvent, dispersing the solution in the carrier and removing the solvent by evaporation to provide the desired solid dispersion. Formulation of Diazepam solid dispersion with different polymer showed in the (table 1-3). $50 \mathrm{mg}$ of Diazepam was taken in the vial and $4 \mathrm{ml}$ acetone was added in each. The drug was completely dissolved in the solvent. Different types of polymer were added in the solution and sonicated (PRO200 homogenizer MIDSCI) it for $1 \mathrm{~min}$.

All solutions were dried by hot air. When the solutions were evaporated completely, they were stored in a dessicator. The formulations were withdrawn from vials, crushed in mortar and pestle, passed through 36 microne sieves. Finally lactose was added in the formulation. Then formulations were transferred in vials and stored in a dessicator. A slightly different approach was warranted for solid dispersions containing poloxamer as it is low melting substance (melting point $52-57^{\circ} \mathrm{C}$ ) and melting method was most suitable for this polymer. The drug was dispersed with methanol in a glass flask and heated in a water bath at $50^{\circ} \mathrm{C}$. Poloxamer was added to the dispersion and it was melted at that temperature. The solvent was evaporated during constant mixing and the resulting solid mass was dried at $40^{\circ} \mathrm{C}$ in hot air oven. The dried samples were crushed in mortar and pestle followed by sieving through 30 mesh screen.

\section{Preparation of standard curve}

10mg Diazepam was accurately weighted and dissolved in $100 \mathrm{ml}$ of Methanol to produce a solution $0.1 \mathrm{mg} / \mathrm{ml} .20 \mathrm{ml}$ of the solution was
Table 1. Formulation of Diazepam solid dispersion by changing the amounts of HPMC 6cps with PEG 6000.

\begin{tabular}{ccccccc}
\hline $\begin{array}{c}\text { Component } \\
\text { (mg) }\end{array}$ & F1 & F2 & F3 & F4 & F5 & F6 \\
\hline Diazepam & 50 & 50 & 50 & 50 & 50 & 50 \\
PEG 6000 & 100 & 100 & 100 & 100 & 100 & 100 \\
HPMC 6cps & 500 & 400 & 300 & 200 & 100 & 0 \\
Lactose & 3 & 3 & 3 & 3 & 3 & 3 \\
\hline
\end{tabular}

Table 2. Formulation of Diazepam solid dispersion by changing amounts of HPC with PEG 6000.

\begin{tabular}{ccccccc}
\hline $\begin{array}{c}\text { Component } \\
\text { (mg) }\end{array}$ & F1 & F2 & F3 & F4 & F5 & F6 \\
\hline Diazepam & 50 & 50 & 50 & 50 & 50 & 50 \\
PEG 6000 & 100 & 100 & 100 & 100 & 100 & 100 \\
HPC & 500 & 400 & 300 & 200 & 100 & 0 \\
Lactose & 3 & 3 & 3 & 3 & 3 & 3 \\
\hline
\end{tabular}

Table 3. Formulation of Diazepam solid dispersion by changing the amounts of Poloxamer 407 with PEG 6000.

\begin{tabular}{ccccccc}
\hline $\begin{array}{c}\text { Component } \\
\text { (mg) }\end{array}$ & F1 & F2 & F3 & F4 & F5 & F6 \\
\hline Diazepam & 50 & 50 & 50 & 50 & 50 & 50 \\
PEG 6000 & 100 & 100 & 100 & 100 & 100 & 100 \\
Poloxamer 407 & 500 & 400 & 300 & 200 & 100 & 0 \\
Lactose & 3 & 3 & 3 & 3 & 3 & 3 \\
\hline
\end{tabular}

transferred in a volumetric flask and volume was made up to $100 \mathrm{ml}$ with distilled water. 1, 2, 3, 4, 5, 6, $7,8,9 \& 10 \mathrm{ml}$ of this solution was taken in $10 \mathrm{ml}$ volumetric flasks and distilled water was added to adjust the volume up to the mark to prepare standard solutions. These serial dilutions were carried out to get different Diazepam concentrations. Standard solutions were then analyzed by UV spectrophotometer (UV-mini-1240, Shimadzu Corp., Kyoto, Japan) at $254 \mathrm{~nm}$ and absorbance was noted. Then the absorbance values were plotted against drug concentration and standard curve of Diazepam was produced (Figure 1).

\section{Quantitative assay}

Formulation containing diazepam was taken in a mortar and triturated properly until fine powder was formed. $5 \mathrm{mg}$ equivalent of fine powder was taken in a $10 \mathrm{ml}$ volumetric flask with the help of a funnel. $5 \mathrm{ml}$ methanol and $5 \mathrm{ml}$ distilled water was added with the powder, sonicated in a sonicator to 


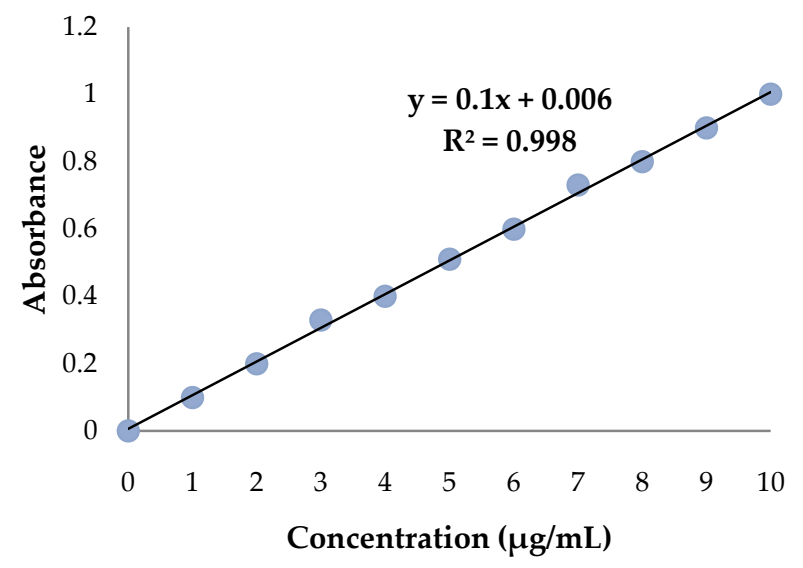

Figure 1. Standard curve of Diazepam in distilled water.

make a clear solution and then finally was filtered. From this solution, $2 \mathrm{ml}$ drug solution was transfeerred in a $100 \mathrm{ml}$ volumetric flask. The volume of the solution was then adjusted up to $100 \mathrm{ml}$ with distilled water.

In the same way, $10 \mathrm{mg}$ equivalent of fine powder was taken in a $25 \mathrm{ml}$ volumetric flask with the help of a funnel. $10 \mathrm{ml}$ methanol and $15 \mathrm{ml}$ distilled water was added with the powder, sonicated in a sonicator to make a clear solution and then finally was filtered. From this solution $2 \mathrm{ml}$ drug solution was withdrawn and taken in a $100 \mathrm{ml}$ volumetric flask. The volume of drug solution was then adjusted up to $100 \mathrm{ml}$ with distilled water. For reagent blank $1 \mathrm{ml}$ methanol was transferred into a $100 \mathrm{ml}$ volumetric flask, diluted to $100 \mathrm{ml}$ with distilled water.

Absorbance value was determined using UVspectrophotometer (UV-mini-1240, Shimadzu Corp., Kyoto, Japan), at $254 \mathrm{~nm}$. Solutions were also diluted if necessary. Using the absorbance value, the amount of Diazepam entrapped was determined with the help of standard curve.

\section{In-vitro release study}

In-vitro dissolution study was performed in a paddle type Dissolution Apparatus (USP Type II Dissolution Apparatus, VEEGO, INDIA). A fixed amount of solid dispersion containing $10 \mathrm{mg}$ equivalent Diazepam from each batch was calculated for dissolution purpose. Distilled water was used as dissolution media. $1000 \mathrm{ml}$ of distilled water was used as dissolution medium in each dissolution basket at a temperature of $37^{\circ} \mathrm{C}$ and a paddle speed of $100 \mathrm{rpm}$. The fixed amount of solid dispersion from each batch was weighed and transferred in each dissolution basket. The dissolution was carried out for 1 hour and $5 \mathrm{ml}$ sample was withdrawn at predetermined intervals of 5, 10, 20,30, 40, 50 \& 60 minutes. Each and every time $5 \mathrm{ml}$ dissolution sample was compensated by another fresh $5 \mathrm{ml}$ distilled water.

Dissolution samples were withdrawn with the help of disposable syringe filter and were kept in a test tube. The dissolution samples were then analyzed spectrophotometrically by UV-VIS spectrophotometer (UV-mini-1240, Shimadzu Corp., Kyoto, Japan) at $254 \mathrm{~nm}$ and absorbance was noted. The dissolution study for each batch was performed in triplicate. These were then analyzed by UV spectrophotometer.

\section{SEM (Scanning Electron microscope) studies}

The surface morphology of the layered sample was examined by using SEM (JSM-6510, JEOL Ltd., Japan). The small amount of powder was manually dispersed onto a carbon tab (double adhesive carbon coated tape) adhered to an aluminum stubs. These sample stubs were coated with a thin layer $(30 \AA)$ of gold by employing POLARON-E 3000 sputter coater. The samples were examined by SEM and photographed under various magnifications with direct data capture of the images onto a computer.

\section{RESULTS AND DISCUSSION}

The effects of three water soluble polymers (HPMC, HPC and poloxamer) on the formulations of Diazepam solid dispersions were compared. The potencies of Diazepam in prepared solid dispersions were analyzed by UV spectrophotometer and the results were within $99-100 \%$ in all cases which indicates uniform mixing of the dispersions.

The in vitro dissolution testing was performed for 45 minutes to ascertain the effect of formulations on immediate drug release enhancement. The enhancement of polymers on drug release from solid dispersion was evaluated by comparing the solubility of drug present in the mixtures as well as of pure drug. Theoretically the solid dispersions improve drug dissolution by decreasing particle size, formation of amorphous forms and improved wettability. 

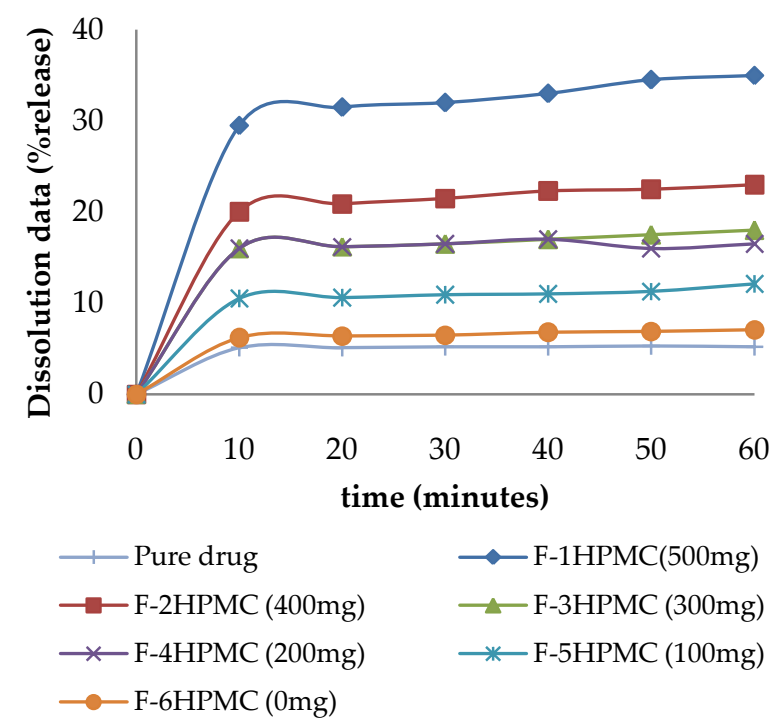

Figure 2. Drug release from HPMC 6 cps formulations.

HPMC show retarded dissolution rate. It is reported that in the case of HPMC solid dispersions, an increase in the amount of polymer up to a certain level resulted in enhanced dissolution rate but further addition of polymer resulted in a decrease of dissolution rate. This finding may be correlated to matrix-forming ability of HPMC. Solid dispersions of hydrophilic swellable polymers such as HPMC become gelatinized in the dissolution medium. HPMC showed retarded drug dissolution, which may be owing to the formation of highly viscous barrier layer at the interface of drug and dissolution medium.

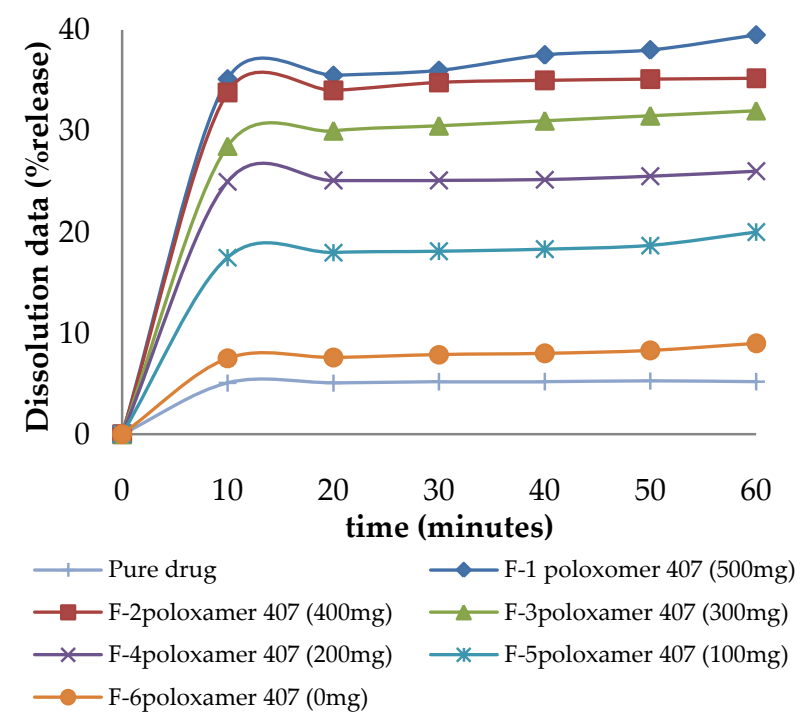

Figure 4. Drug release from Poloxamer 407 formulations.

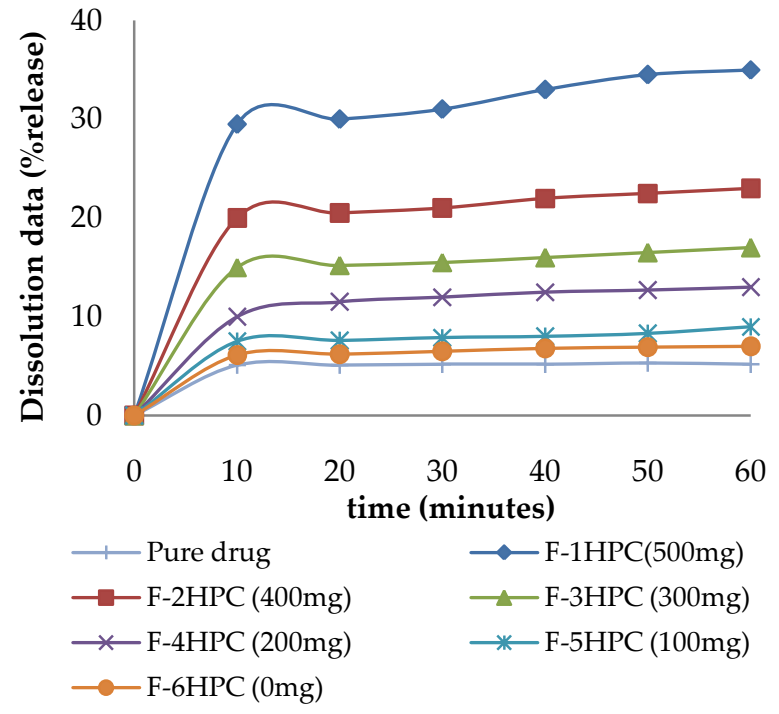

Figure 3. Drug release from HPC formulations.

The better dissolution rate of solid dispersion may be due to an improved wettability of drug particles, a significant reduction in particle size during formation of solid dispersion and dissolution due to increase in the surface area of drug, proper dispersion and increase in the amorphicity of drug by adsorption on the surface of adsorbent. High rate of dissolution of soluble polymer component of solid dispersion which would pull along more insoluble but finely mixed drug into dissolution medium. Similar studies was performed by (Hsiu-O et al., 1996) the dissolution rate of nifedipine increased as more HPMC was added to the solid dispersions.

From the curve (Figure 2) it is observed that increases the HPMC with other polymer were fixed and the drug release was increase significantly. In the case of pure drug, release was decreased than drug with the combination of other polymer. When HPMC is increased drug release from solid dispersion increase significantly due to wetting ability and convert crystalline to amorphous. The formulation containing increased HPMC increased drug release.

The drug release was reduced by higher amount of HPMC. The polymer, when used at high concentration, forms a gelatinous layer around drug particles upon contact with aqueous media and which acts as a barrier to drug release. The drug is released slowly from such matrix by diffusion process. Usually the higher molecular weight HPMC (such as HPMC $\mathrm{K} 15, \mathrm{~K} 100)$ are used for sustained release effect in 

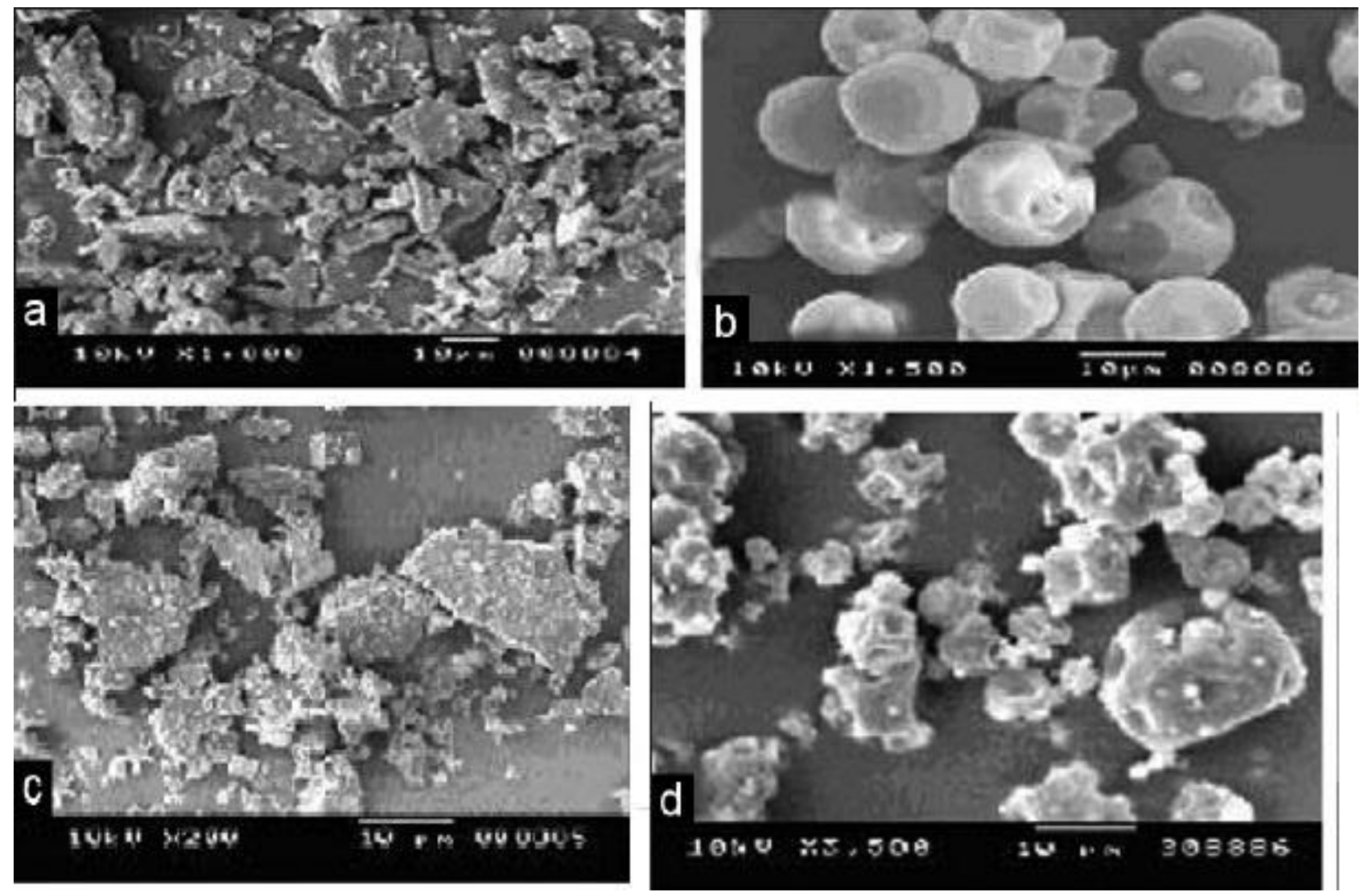

Figure 5. SEM pictures of the drug and formulations. (a) Pure drug, (b) Solid dispersion HPMC, (c) Solid dispersion HPC, (d) Solid dispersion Poloxamer

tablet formulations but in case of solid dispersions the low molecular weights are capable of achieving the enhance drug release.

The formulation containing 1:10 drug-polymer, the drug release was highest due to wetting ability (Figure 2). At high concentration HPMC provides wetting of drug and improves dissolution. It is supported by the fact that increased drug release was observed with formulations containing drug/polymer 1:10 ratio (Figure 2). This formulation contained small amount of crystal lattice compared to pure drug alone which indicates reduction of drug particle size may be responsible for improved dissolution.

From the curve (Figure 3) it is observed that increases the HPC with other polymer were fixed and the drug release was increase significantly. In the case of pure drug, release was decreased than drug with the combination of other polymer. When HPC is increased drug release from solid dispersion increase significantly due to wetting ability and convert crystalline to amorphous. The formulation containing increased HPC increased drug release.

From the curve (Figure 4) it is observed that increases the Poloxamer with other polymer were fixed and the drug release was increase significantly. In the case of pure drug, release was decreased than drug with the combination of other polymer. When poloxamer is increased drug release from solid dispersion increase significantly due to wetting ability and convert amorphous to crystalline. The formulation containing increased poloxamer increased drug release.

Poloxamer 407 can act as gelling agent at high concentration and it can affect drug dissolution from solid dispersions. Drug release was very rapid compared to other polymers ratios. But similar to HPMC, higher amount of poloxamer facilitated drug release. When the poloxamer concentration was decreased the drug release was also decreased. 
Although dissolution rate for poorly water-soluble drug can be enhanced by converting the drug into its amorphous form, but it can be thermodynamically unstable, and under certain levels of heat and humidity, could recrystallize into a more stable, poorly water-soluble form. Diazepam could be crystallized out of the formulations from poloxamer containing solid dispersions after contact with aqueous media. Poloxamers rapidly dissolved away from the solid dispersions and amorphous Diazepam reverted back to its crystalline state.

It was reported that molecular dispersion is one of the important roles of drug release from the polymer-drug system. The present work shows that the dissolution rate of Diazepam from solid dispersions with PEG 6000 and poloxamer improved compared to the pure drug. Further, solid dispersions performed better than the corresponding physical mixtures. Various studies have shown that freely water soluble carriers inhibit crystallization of drugs in solid dispersions resulting in amorphous form of the drug in the solid dispersions. Crystallization inhibition is attributed to two effects: interactions, such as hydrogen bonding between the drug and the polymer and the entrapment of the drug molecules in the polymer matrix during solvent evaporation or a combination of both. The present study confirmed the advantage of improved aqueous solubility of Diazepam in its solid dispersions form, which can be formulated as tablets with better dissolution characteristics.

\section{SEM study of solid dispersion}

SEM study indicated (Figure 5) that pure drug particles were irregular in shape, while the physical mixture of the drug and carrier shows that drug particle remains dispersed and physically adsorbed on the surface of the carrier particles. The solid dispersion of Diazepam and HPMC 6cps showed a homogeneous dispersion indicating that the diazepam molecules were dispersed uniformly in carrier matrices of solid dispersion prepared solvent evaporation method, assuming amorphous solid dispersion state.

\section{CONCLUSION}

Solid dispersion is proven to be a useful technique to improve the solubility of poorly soluble drugs like Diazepam. Solid dispersions prepared from hydrophilic polymers using the solvent evaporation method were effective in improving drug dissolution. The dispersion containing Poloxamer (Table 2.) shows acceptable dissolution compared to the HPMC, HPC dispersion or pure drug. The study revealed that optimum levels of hydrophilic carriers and hydrophillic porous adsorbents ensure a prompt and complete dissolution of Diazepam from solid dispersions that are used in oral pharmaceutical formulations. Depending on experimental data it is concluded that the type of polymer and drugpolymer ratio are the critical factors for the development of solid dispersions.

\section{ACKNOWLEDGEMENT}

The authors are thankful to Rangs Pharmaceuticals Ltd., Dhaka, Bangladesh for their generous donation of Diazepam. The authors are also thankful to Northern University Bangladesh and the University of Dhaka for their supports and co-operations.

\section{REFERENCES}

Akhter and Hossain,(2012) Dissolution enhancement of Capmul PG8 and Cremophor EL based Ibuprofen Self Emulsifying Drug Delivery System (SEDDS) using Response surface methodology International Current Pharmaceutical Journal;1(6): 138-150. [DOI]

Chen Y., Zhang, GGZ., Neilly, J., Marsh, K., Mawhinney, D., Sanzgiri, YD.( 2004). Enhancing the bioavailability of ABT963 using solid dispersion containing pluronic F-68. Int. J.Pharm; 286: 69-80. [DOI]

Chiou, W.L., Rigelman, S.( 1971). Pharmaceutical application of solid dispersion system. J Pharm Sci.; 60: 1281-1302. [DOI]

Chokshi, RJ., Zia, H., Sandhu, HK., Shah, NH., Malick, WA.( 2007). Improving the Dissolution Rate of Poorly Water Soluble Drug by Solid Dispersion and Solid Solution-Pros and Cons. Drug Delivery, 14: 33-45. [DOI]

Costantino, HR., Firouzabadian, L. Wu. C., Carrasquillo, KG., Griebenow, K., Zale SE., Tracy, MA.(2002). Protein spray freeze drying. 2. Effect of formulation variables on particle size and stability. J. Pharm. Sci; 91, 2: 388-395. [DOI]

Douroumis, D., Bouropoulos, N., Fahr, A.( 2007). Physicochemical characterization of solid dispersions of three 
antiepileptic drugs prepared by solvent evaporation method. J Pharm. Pharmacol.; 59: 645-653. [DOI]

Gao, P., Rush, BD., Pfund, WP., Huang, T., Bauer, JM., Morozowich, W., Kuo, MS., Hageman, MJ. (2003). Development of a supersaturable SEDDS (S-SEDDS) formulation of paclitaxel with improved oral bioavailability. J. Pharm. Sci., 92, 12: 2386-98. [DOI]

Gohel, M. C., Patel, L. D.(2002). Improvement of nimesulide dissolution from solid dispersions containing crosscarmellose sodium and Aerosil ${ }^{\circledR} 200$. Acta Pharm.;52: 227-241.

Hancock, BC., Parks, M.(2000). What is the true solubility advantage for amorphous pharmaceuticals? Pharm. Res; 17: 397-404. [DOI]

Hsiu-O, Ho. , Huei-Lin, Su., Tsuimin, Tsai., Ming-Thau, Sheu.(1996). The preparation and characterization of solid dispersions on pellets using a fluidized-bed system. International Journal of Pharmaceutics.; 139: 1-2, 223-229. [DOI]

Iervolino, M., Cappello, B., Raghavan, SL., Hadgraft.(2001) . Penetration enhancement of Ibuprofen from supersaturated solutions through human skin. Int. J Pharm.; 212: 131-41. [DOI]

Konno, H., Handa, T., Alonzo, DE., Taylor, LS. (2008). Effect of polymer type on the dissolution profile of amorphous solid dispersions containing felodipine. Eur. J Pharm. Biopharm., 70, 2: 493-9. [DOI]

KPR, Murthy, KVR., Hayman, AR., Becket, G.( 2003). Physicochemical characterization of nimesulide- cyclodextrin binary systems. AAPS Pharm.Sci.Tech.;41, 7:1-12.

Lehoullier, PF., Ticku, MK.(1987). Benzodiazepine and betacarboline modulation of GABA-stimulated 36Cl-influx in cultured spinal cord neurons.Eur J Pharmacol ; 135(2):235238. [DOI]

Leuner, C., Dressman J. (2000) . Improving drug solubility for oral delivery using solid dispersions. Eur J Pharm Biopharm.; 50: 47-60. [DOI]

Lipp, R.(1998). Selection and use of crystallization inhibitors for matrix-type transdermal drug-delivery systems containing sex steroids. J. Pharm. Pharmacol.; 50, 12: 1343-9. [DOI]

Matsuda, Y., Kawaguchi, S.(1986). Physicochemical characterization of oxyphenbutazone and solid-state stability of its amorphous form under various temperature and humidity conditions. Chem. Pharm. Bull; 34:1289-1298. [DOI]

Nair, R., Gonen, S., Hoag, SW.(2002). Influence of polyethylene glycol and povidone on the polymorphic transformation and solubility of carbamazepine. Int. J Pharm.;240: 11-22. [DOI]

Newa, M., Bhandari, KH., Oh, DH., Kim, YR., Sung, JH., Kim, JO., Woo, JS., Choi, HG., Yong, CS.(2008). Enhanced Dissolution of Ibuprofen Using Solid Dispersion with Poloxamer 407. Arch Pharm. Res; 31, 11: 1497-1507. [DOI]
Passerini, N., Gonzalez-Rodriguez, ML., Cavallari, C., Rodriguez, L., Albertini, B.(2002). Preparation and characterization of ibuprofen-poloxamer 188 granules obtained by melt granulation. Eur. J Pharm. Sci.; 15: 71-78. [DOI]

Proud foot, S.(1991).Factors affecting bioavailability: factors influencing drug absorption from the gastrointestinal tract. In: Aulton ME, editor. Pharmaceutics: the science of dosage from design. Edinburgh: Churchill Livingstone; pp. 135-173.

Rane, Y., Mashru, R., Sankalia, M., Sankalia, J.(2007). Effect of Hydrophilic Swellable Polymers on Dissolution Enhancement of Carbamazepine Solid Dispersions Studied Using Response Surface Methodology. AAPS Pharm.SciTech.;8:27 [DOI]

Shah, TJ., Amin, AF., Parikh, JR., Parikh, RH..(2007). Process Optimization and Characterization of Poloxamer Solid Dispersions of a Poorly Water-soluble Drug. AAPS PharmSciT.ech ; 8, 2: Article 29. [DOI]

Skerritt, JH., Johnston, GA.(983).Enhancement of GABA binding by benzodiazepines and related anxiolytics. Eur. J. Pharmacol. 1;89: 3-4, 193-198. [DOI]

PD Thahera1, Ashok, K Latha, T Shailaja, S Nyamathulla, MU Uhumwangho.(2012). Formulation and evaluation of Norfloxacin gastro retentive drug delivery systems using natural polymers. International Current Pharmaceutical Journal , 1(7): 155-164 [DOI]

United States Pharmacopoeia, 23, NF 18. The USP Convention, Rockville 1995; pp. 1791-1799.

Van, DMG., Augustijns, P., Blaton, N., Kinget, R. (1998) Physicochemical characterization of solid dispersions of temazepam with polyethylene glycol 6000 and PVP K30. Int. J. Pharm.; 164: 67-80. [DOI]

Yamashita, K., Nakate, T., Okimoto, K., Ohike, A. (2003). Establishment of new preparation method for solid dispersion formulation of tacrolimus. Int. J. Pharm.;267: 79-91. [DOI]

Yh-Nam, P., Jing-Huey, C., Russel, R.C.et al.(2000). Enhancement of dissolution and bioavailability of piroxicam in solid dispersions systems. Drug Dev. Ind. Pharm.;26:989-994. 